\title{
The effects of antioxidants and shelf life conditions on oxidation markers in a sunflower oil salad dressing emulsion (SOSDE)
}

Jeanine Sainsbury ${ }^{1,2}$, Roman Grypa ${ }^{2}$, John Ellingworth ${ }^{2}$, Kwaku G Duodu ${ }^{1}$, Henriëtta L De Kock $^{1}$

${ }^{1}$ Department of Food Science, University of Pretoria, Pretoria Private Bag X20, Hatfield 0002, South Africa

${ }^{2}$ McCormick \& Company Inc., 204 Wight Avenue, Hunt Valley, MD 21030, USA

*Corresponding author: Henriëtta L de Kock, riette.dekock@up.ac.za

\section{Highlights}

- An accelerated storage model was applied to a sunflower oil-in-water emulsion (SOSDE).

- SOSDEs in accelerated conditions did not clearly predict deterioration of ambient stored.

- The model is more suitable for SOSDEs with EDTA than gallic acid as antioxidant.

- Peroxide and anisidine values were more sensitive oxidation markers for the storage model.

\begin{abstract}
The effects of levels of antioxidant [gallic acid or ethylene diamine tetraacetate (EDTA)] in a sunflower oil salad dressing emulsion (SOSDE) and shelf life affecting conditions on aroma, anisidine values (AV) and peroxide values (PV) were determined. Aroma differences between products with different concentrations of antioxidants were more apparent for ambient than accelerated stored SOSDEs. Aroma differences were more noted between SOSDEs with different antioxidants than antioxidant concentrations per se. PV differences between
\end{abstract}


accelerated stored SOSDEs with high and low EDTA concentrations were found. AV differences existed between SOSDEs with different gallic acid concentrations for both storage conditions, and for accelerated stored SOSDEs with different EDTA concentrations. The accelerated storage model is more suitable for SOSDEs with metal chelator antioxidants e.g. EDTA, than free radical scavenging antioxidants e.g. gallic acid. PV, AV and aroma of accelerated stored SOSDEs do not clearly predict ambient shelf life.

\section{Keywords}

Rancid, Lipid oxidation, Sensory, Antioxidant, Pro-oxidant, Accelerated shelf life, Emulsion

\section{INTRODUCTION}

Consumers demand high-quality shelf stable products with shelf lives of several weeks or months (Corrigan, Hedderley \& Harvey, 2012). Sunflower oil emulsions, like salad dressings and mayonnaise, have a shelf life of 6-12 months at room temperature. The shelf life of these products is often determined by quality changes rather than microbial safety of the product (Corrigan et al., 2012; Lewis \& Heppel, 2000). Spoilage of emulsions is related to lipid oxidation or deterioration of the emulsifying agent that lead to oil separation (Bou et al., 2011; Rossel, 1999). Antioxidants retard lipid oxidation and the formation of rancid off notes in emulsions (Rodríguez-Rojo, Visentin, Maestri \& Cocero, 2012). Lipid oxidation or rancidity is the term used to describe the complex sequence of chemical interactions in oils where the fatty acids react with oxygen to form volatile aldehydes, ketones, alcohols, furans, hydrocarbons or acids. These volatile compounds and combinations thereof are associated with the rancid off aroma (Hamilton, 1999) which can be analysed using a variety of chemical and sensory tests. Flavour release of volatiles is strongly affected by the food matrix (Hartvigsen, Lund, Hansen, \& 
Holmer, 2000). The efficacy of natural antioxidants like tocopherol and rosemary extract (Rodríguez-Rojo et al., 2012) has been researched extensively due to potential toxicological long term effects of synthetic antioxidants such as butylated hydroxyanisole (BHA) and tertbutylhydroquinone (TBHQ) (Asnaashari, Farhoosh, \& Sharif, 2014; Rodríguez-Rojo et al., 2012).

Accelerated shelf life tests are often used to determine shelf life due to time and cost constraints associated with real time shelf life tests (Corrigan et al., 2012). During accelerated shelf life tests, the product is exposed to factors or combinations thereof that promote quality deterioration eg. higher temperatures, above normal humidity and light or specifically UV light exposure, higher water activity and/or addition of pro-oxidants. Acceleration factors depend on the product and normal storage conditions for the product (Richards, De Kock, \& Buys, 2014). Most studies have evaluated the effects of one factor at a time (Branco, Rodrigues, Gioielli, \& Castro, 2011). For example the Arrhenius equation has been used to relate temperature of storage with reaction velocity (Córdova, Quezada, \& Saavedra, 2011). However shelf life is a dynamic process in which storage factors constantly interact with each other (Branco et al., 2011). Therefore accelerated shelf life testing would be more accurate and predictive if the contributing factors could be evaluated simultaneously (Branco et al., 2011).

The objective of this study was to determine the effect of different concentrations of the natural antioxidant, gallic acid and the synthetic antioxidant, ethylene diamine tetraacetate (EDTA) on orthonasally perceived aroma attributes, anisidine value (AV) and peroxide value (PV) of a sunflower oil-in-water salad dressing emulsion (SOSDE). Emulsions were stored at ambient shelf conditions as well as at accelerated shelf life conditions as proposed by Branco et al., (2011) who developed an accelerated shelf life model for oil-in-water emulsions to optimize conditions to evaluate natural antioxidants. Branco et al (2011) applied this accelerated shelf life model to a model oil-in-water emulsion. What is novel here is the application of the Branco accelerated shelf life model to evaluate antioxidant effects in a commercial SOSDE emulsion formulation and to compare with the shelf life also under ambient conditions. Oxidative stability 
of emulsions influenced by antioxidant levels and shelf life affecting conditions can potentially be better understood and predicted by development of this new technique

\section{MATERIALS AND METHODS}

The shelf life model as proposed by (Branco et al., 2011) with modification to timing intervals of analyses was used for the accelerated study component.

\subsection{Preparation of emulsions}

Table 1 shows the experimental design for the storage studies conducted under ambient and accelerated oxidative conditions.

Table 1: Preparation and storage conditions for the 10 sunflower oil salad dressing emulsions (SOSDEs)

\begin{tabular}{|c|c|c|c|c|c|c|c|c|c|c|}
\hline & \multicolumn{10}{|c|}{ Sunflower oil salad dressing emulsions (SOSDE) } \\
\hline Products & $\begin{array}{l}\text { 을 } \\
\text { 은 }\end{array}$ & 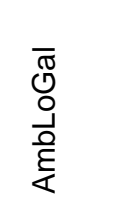 & $\begin{array}{l}\overline{\mathbb{N}} \\
\frac{0}{T} \\
\frac{0}{E} \\
\frac{1}{4}\end{array}$ & 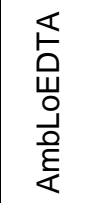 & 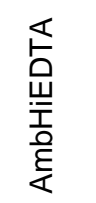 & 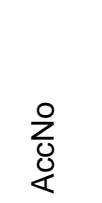 & $\begin{array}{l}\bar{\pi} \\
00 \\
\\
0 \\
\end{array}$ & $\begin{array}{l}\overline{\mathbb{N}} \\
\stackrel{0}{\bar{T}} \\
\frac{0}{0}\end{array}$ & 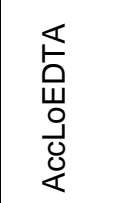 & 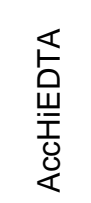 \\
\hline Treatment & \multicolumn{5}{|c|}{ Ambient } & \multicolumn{5}{|c|}{ Accelerated } \\
\hline $\begin{array}{lr}\text { Pro-oxidants } & \text { added } \\
(0.885 \mathrm{mmol} / \mathrm{L} & \mathrm{FeSO}_{4} \text {, } \\
1.70 \mathrm{mmmol} / \mathrm{L} & \text { Ascorbic } \\
\text { acid). pH 5.5 } & \end{array}$ & \multicolumn{5}{|c|}{ No } & \multicolumn{5}{|c|}{ Yes } \\
\hline Antioxidant & None & \multicolumn{2}{|c|}{ Gallic Acid } & \multicolumn{2}{|c|}{ EDTA } & None & \multicolumn{2}{|c|}{ Gallic Acid } & \multicolumn{2}{|c|}{ EDTA } \\
\hline Antioxidants added & None & $500 \mathrm{ppm}$ & $\begin{array}{l}1000 \\
\mathrm{ppm}\end{array}$ & $\begin{array}{l}37.5 \\
\mathrm{ppm}\end{array}$ & $75 p p m$ & None & $500 \mathrm{ppm}$ & $\begin{array}{l}1000 \\
\mathrm{ppm}\end{array}$ & $37.5 p p m$ & $75 \mathrm{ppm}$ \\
\hline $\begin{array}{l}\text { Frequency of aroma } \\
\text { analysis }\end{array}$ & \multicolumn{5}{|c|}{ Bi-weekly } & \multicolumn{5}{|c|}{ Daily } \\
\hline $\begin{array}{l}\text { Frequency of chemical } \\
\text { analysis }\end{array}$ & \multicolumn{5}{|c|}{ Week $0,2,4,8,12,16,20$ and 24} & \multicolumn{5}{|c|}{ Day $0,1,2,4,6,8,11,14,17$ and 20} \\
\hline Storage temperature & \multicolumn{5}{|c|}{$25^{\circ} \mathrm{C}$} & \multicolumn{5}{|c|}{$32.2^{\circ} \mathrm{C}$} \\
\hline Storage period & \multicolumn{5}{|c|}{24 weeks } & \multicolumn{5}{|c|}{20 days } \\
\hline
\end{tabular}

Oil-in-water emulsions (8.5\%) were prepared. The ingredients for the emulsions consisted of sunflower oil, water, white sugar, vinegar, egg yolk powder, stabilisers, salt, mustard powder and black pepper. The dry ingredients consisting of sodium benzoate $(2.39 \mathrm{~g})$, potassium sorbate $(2.39 \mathrm{~g})$, ascorbic acid $(2.39 \mathrm{~g} 1.70 \mathrm{mmol} / \mathrm{L}), \mathrm{FeSO}_{4}(0.86 \mathrm{~g} 0.885 \mathrm{mmol} / \mathrm{L})$ 
and antioxidants (gallic acid or EDTA) were dissolved in water $(600 \mathrm{~g})$ by mixing the solution for 2 minutes. Salt (79.8 g), white sugar (997.9 g), mustard powder (47.9 g), ground black pepper $(0.8 \mathrm{~g})$ and egg yolk powder (159.7 $\mathrm{g}$ ) were mixed into the solution. Additional water (2813.2 $\mathrm{g}$ ) was added. $\mathrm{NaOH}(673 \mathrm{~g} 0.1 \mathrm{M})$ was only added to products that were stored in accelerated storage conditions. The quantity of $0.1 \mathrm{M} \mathrm{NaOH}$ added to alter the $\mathrm{pH}$ to 5.5 was determined in a pre-study. After the addition of $\mathrm{NaOH}$ and water, the mixture was mixed for 5 minutes. Separately sunflower oil $(479 \mathrm{~g})$ was mixed with the stabiliser Ultra Tex $(199.6 \mathrm{~g})$ obtained from Ingredion Inc. (Westchester, IL, USA) and Visco Xan (8.8 g) obtained from Archer Daniels Midland (Decatur, IL, USA) for 3 minutes and then left to rest for 5 minutes. After the resting period, the solution of sunflower oil and stabilisers was added to the initial solution of dry ingredients and mixed for 2 minutes. Thereafter additional, sunflower oil $(117.7 \mathrm{~g})$ was gradually added while mixing continued. Lemon concentrate $(4.0 \mathrm{~g})$, vinegar $(780.8 \mathrm{~g})(10 \%)$ and lactic acid $(16 \mathrm{~g})$ were added to the solution and mixed for 5 minutes. Accelerated stored products contained pro-oxidants as proposed by (Branco et al., 2011) for optimal accelerated lipid oxidation. The products contained either no antioxidants (No) or low or high levels of gallic acid (LoGal and HiGal) or EDTA (LoEDTA or HiEDTA) as per Table 1.

All products were prepared using the same batch of raw materials and using the same equipment. The $\mathrm{pH}$ of each emulsion was measured ( $\mathrm{pH}$ 3.24-3.32) and the $\mathrm{pH}$ of accelerated products was adjusted to 5.50 using $0.01 \mathrm{M} \mathrm{HCl}$ or $0.1 \mathrm{M} \mathrm{NaOH}$, and then homogenised using a Scott Turbon Mixer (LA1.3, Adelanto, California. USA). Each emulsion was poured into several clear glass containers $(80 \mathrm{ml}$ emulsion per $100 \mathrm{ml}$ container). Ambient shelf life SOSDEs (stored at $25^{\circ} \mathrm{C}$ ) were collected every 2 weeks (14 days) and analysed for aroma properties, PV and AV. Accelerated shelf life products (stored at $32.2^{\circ} \mathrm{C}$ ) were collected every $24 \mathrm{~h}$ and analysed for aroma properties, PV and AV. Selected glass bottles were stored in a freezer at - 
$18.9^{\circ} \mathrm{C}$ until time of evaluation. Bottles were removed from the freezer $4 \mathrm{~h}$ before analyses to reach a temperature of $22-24^{\circ} \mathrm{C}$.

\subsection{Evaluation of the aroma of SOSDEs}

Sensory evaluation was performed by 12 trained sensory panellists whose selection was based on their performance in screening tests. The screening tests included recognition of rancidity and rancidity related aromas such as cardboard, plastic and paint (Jacobsen, 1999) as well as other general aroma attributes (e.g. pungency, eggy and dairy aromas) in oil-in-water emulsions. As an initial guideline for this study, attributes, references and definitions from a previous study on lipid oxidation (Arancibia, Jublot, Costell, \& Bayarri, 2011) were used. During four orientation sessions ( $2 \mathrm{~h}$ each), panellists received each of the five SOSDE products and determined which attributes best described the differences among the SOSDEs. Due to potential health implications associated with the consumption of oxidised food products (Chen, McClements, \& Decker, 2011) the panel evaluated orthonasally perceived aroma attributes only. Lerma-García et al., (2010) conducted research on defects in oils and noted that the most important properties of oil are represented by their aroma. Clear definitions and references were finalised for the 13 aroma attributes evaluated (Table 2).

Evaluation was conducted under red light to mask any colour differences between products (Meilgaard, Civille, \& Carr, 2007). Five samples were analysed per day with 5 minute breaks between samples. Panellists spent as long as they needed on a sample. They were instructed to smell their clean hand palms in between sniffs. All products were evaluated in duplicate. All data recording was completed on computer using Compusense Five version 5.2 (Compusense Inc., Guelph, Canada). Products $(80 \mathrm{ml}$ ) were served in $100 \mathrm{ml}$ glass containers with lids, labelled with random 3-digit codes. Due to low detection levels of rancidity related attributes expected in products and the complexity of aroma evaluation, panellists had to merely indicate the presence or absence of an attribute rather than rating the intensity of the attribute. 
In order to ensure that panellists were not influenced in any way, no information regarding the nature of the experiment (shelf life testing) was provided.

Table 2: Definitions for attributes developed in the aroma evaluation of sunflower oil salad dressing emulsions (SOSDE)

\begin{tabular}{ll}
\hline Aroma Attributes & Definition \\
\hline Pungent & $\begin{array}{l}\text { Sharp, piercing, somewhat irritant odour that gives a recoil response. } \\
\text { Tactile stinging sensation. }\end{array}$ \\
\hline Vinegar & Aroma associated with white vinegar \\
\hline Eggy & Aroma associated with boiled egg. Sulphur like. \\
\hline Citrus & Aroma associated with acidic fruity notes of the citrus family \\
\hline Musty & $\begin{array}{l}\text { Aroma associated with attic smell, mouldy, damp, reminiscent of air in an } \\
\text { unventilated room. }\end{array}$ \\
\hline Dairy & Aroma associated with non-fermented dairy products such as cow's milk \\
\hline Green & Aroma associated with freshly cut leaves, grass or green vegetables. \\
\hline Oil (fresh) & Aroma associated with fresh oil \\
\hline Earthy & Aroma associated with moistened soil \\
\hline Metallic & Aroma associated with metals \\
\hline Plastic & Aroma associated with a waxy oxidised off note (associated with a plastic \\
container)
\end{tabular}

\subsection{Chemical analysis of SOSDEs}

To measure the lipid hydroperoxide concentrations (peroxide values, PV) of SOSDEs at each storage period (Table 1), the peroxide method (IDF74A: 1991), described by Shanta \& Decker (1994) was followed. The p-anisidine values (AV) of the products were determined in duplicate according to the AOCS Official Method Cd 18-90 (AOCS, 1999).

\subsection{Statistical analyses}

The detection of aroma attributes in the SOSDE products was expressed as a binomial function of the proportion of the panellists who detected the attribute. Statistical analysis was conducted using GenStat ${ }^{\circledR}$. The generalized linear mixed model (Payne, 2012) analysis was 
applied to the sensory data with the binomial distribution and logit link function. The fixed effects were specified as products and storage period (days or weeks) and the random effect as replication. Mean proportions of presence/absence (between 0 and 1) were separated with Fisher's protected least significant difference (FPLSD) test at the $5 \%$ level. The linear mixed model analysis, or REML, was applied to chemical analyses (Payne, 2012). The fixed effects were specified as products, days and the products $\mathrm{x}$ days interaction. FPLSD test was used to compare means at the $1 \%$ level as the treatment variances were heterogeneous. If variances are unequal, this can affect the Type I error rate and therefore a more strict decision criteria needs to be applied (Zimmerman, 2000). Separate analyses were conducted for ambient and accelerated stored SOSDEs.

\section{RESULTS}

\subsection{Sensory and chemical analyses of SOSDEs with different levels of antioxidants and stored under ambient shelf life conditions}

The effects of no antioxidant and low and high concentrations of antioxidants on perception of aroma attributes of SOSDEs stored at ambient storage are shown in Figure 1. There was no significant difference in pungent or green aromas between SOSDEs with high and low concentrations of either antioxidant, however differences in musty were apparent when high and low concentration of both antioxidants were applied to the SOSDE. For SOSDEs with EDTA, vinegar aroma was significantly more noted at the higher concentration. Citrus aroma was less noted in AmbHiGal, while differences were not noted for EDTA treated SOSDEs. Oil aroma in AmbHiEDTA was detected by $10 \%$ fewer panellists compared to AmbLoEDTA. Although the oil aroma attribute was not associated with rancidity during training sessions, oil and fried oil are sensory terms often used to describe the formation of aldehydes such as benzene acetaldehyde, trans,trans-2,4-octadienal, trans,cis-2,4 nonadienal, trans,trans-2,4- 
nonadienal, trans,cis-2,4-decadienal, trans,trans-2,4-decadienal (Hartvigsen et al., 2000). It is possible that the perceived oil aroma was due to the presence of these aldehydes during oxidation and may therefore be an indication of lipid oxidation, but further tests would be needed to confirm the specific aldehydes in the SOSDE. Of the oxidation related aroma attributes; plastic, painty, cardboard and rancid (Hudson \& Gordon, 1999), rancid was the only attribute that showed differences between SOSDEs with high and low concentrations of antioxidants (Fig 1). Rancid aroma was detected by a significantly higher percentage of panellists in AmbLoGal compared to AmbHiGal. In contrast, rancid aroma was detected by significantly fewer panellists in AmbLoEDTA compared to AmbHiEDTA. No significant effect in cardboard and painty aroma was detected among the SOSDEs with no antioxidant and gallic acid, indicating the minimal effect of low and high concentrations of gallic acid on retarding development of oxidation related aroma attributes.

Upon comparison of different antioxidants, EDTA applied to the SOSDE decreased the presence of earthy and metallic aromas compared to SOSDEs containing no antioxidant and gallic acid, and EDTA respectively (Fig 1). In shelf life studies of emulsions it was found that these two sensory attributes (earthy and metallic) could be related to the development of ketone 1-hexen-3-one during oxidation (Hartvigsen et al., 2000). However, according to Jacobsen (1999) it is impossible to conclude which volatiles relate to which descriptor without testing specifically. Further analysis is needed in order to determine the presence of 1-hexen-3one and its relation to earthy and metallic aroma in the SOSDE. 


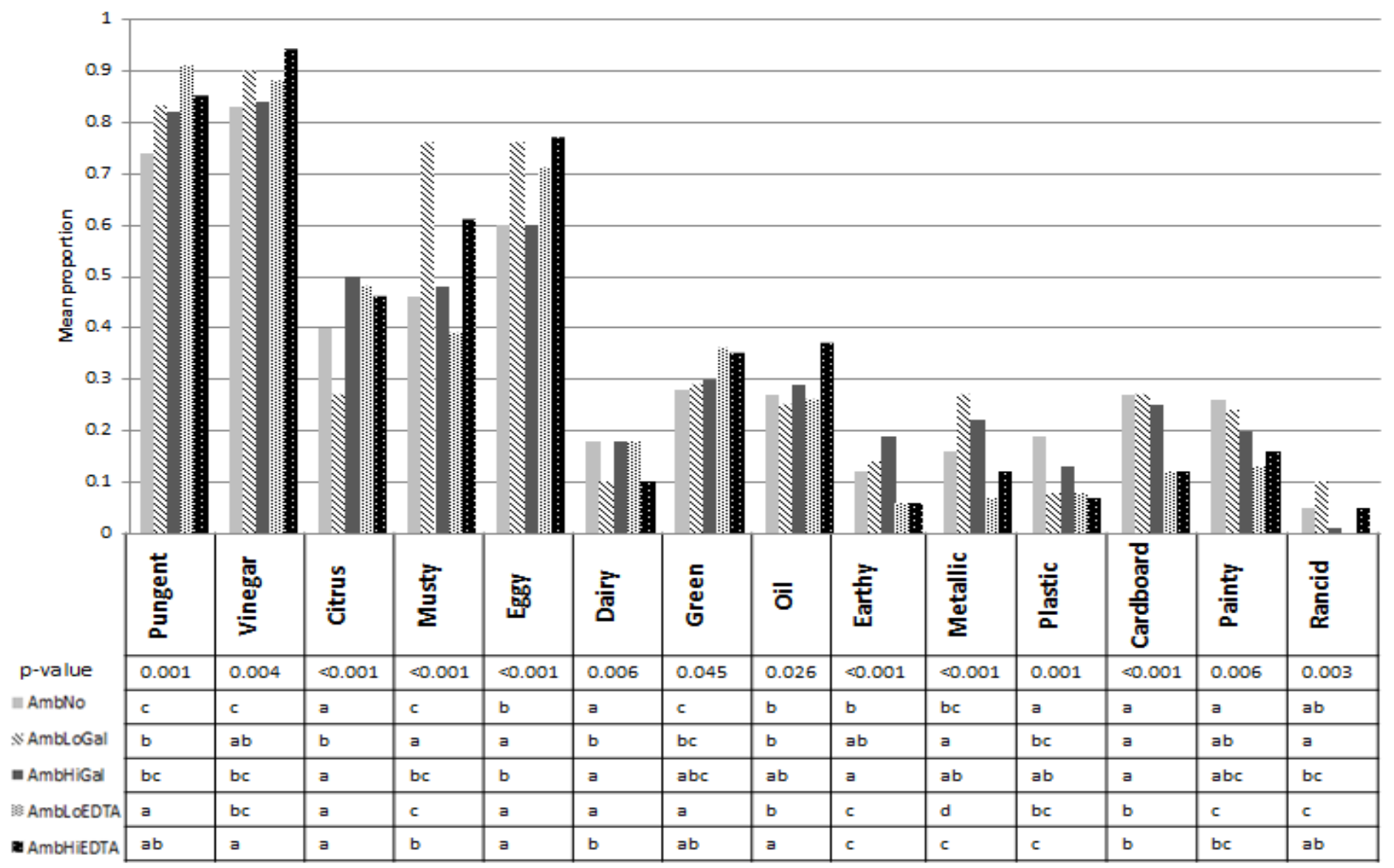

abc - for a specific aroma attribute, products with different letters were significantly different at the $95 \%$ confidence level

Fig 1. The effect of low and high concentrations of antioxidants or none added on aroma attributes for SOSDEs stored at ambient conditions for 24 weeks with measurements taken every 2 weeks (see Table 1 for detail of product code names).

To better understand how different concentrations of the antioxidants gallic acid and EDTA influenced oxidation of SOSDE, PV and AV were determined for each SOSDE during ambient storage (Fig 2i). Lipid peroxide formation, measured by $\mathrm{PV}$, was not affected by different concentrations of different antioxidants $(p=0.042$, Fig 2i). Alamed, McClements and Decker (2006) reported research on emulsions with different concentrations of EDTA, and found that higher concentrations of EDTA retarded lipid oxidation over longer periods of time. Similarly Bou et al. (2011) found that increasing concentrations of free radical scavenging antioxidants (gallic acid and propyl gallate) decreased lipid oxidation, measured through relative lycopene 


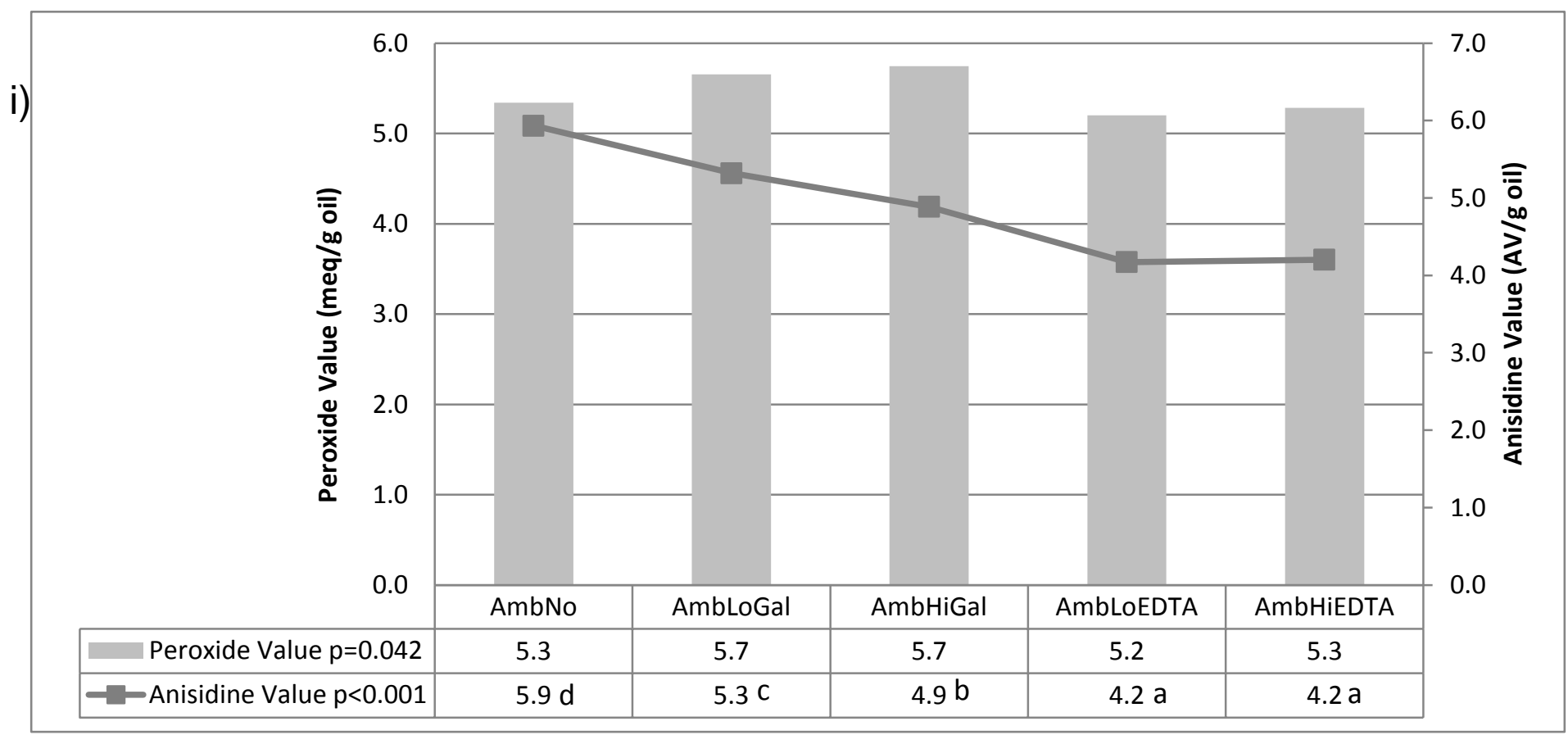

abcd - values in a row with different letters indicate significant differences at $90 \%$ confidence level

ii)

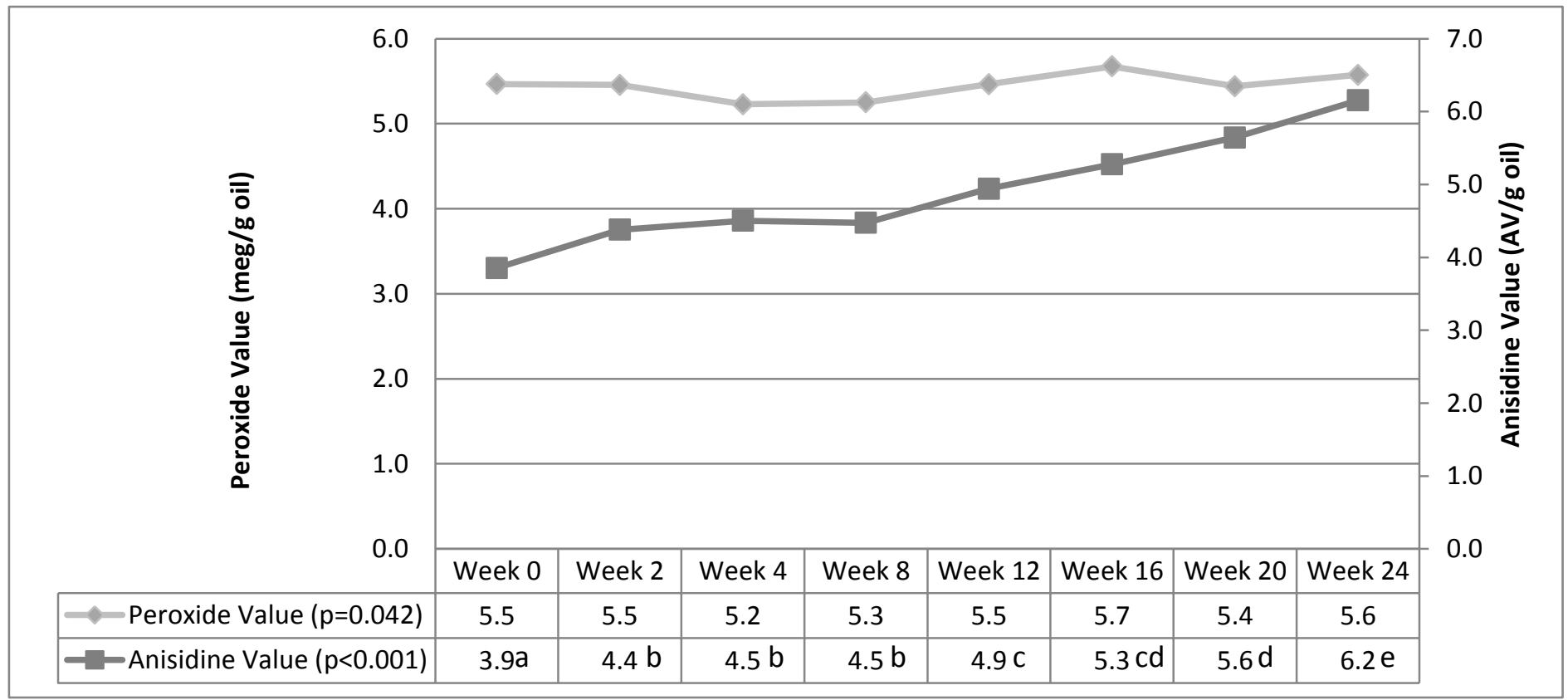

abcde - values in a row with different letters indicate significant differences at $99 \%$ confidence level

Fig 2. Chemical analyses (Peroxide Value and Anisidine Value) for oil extracted from SOSDEs stored under ambient shelf life conditions illustrated for (i) different concentrations of EDTA and gallic acid antioxidants and (ii) 24 week time period. 
concentrations over time. This was in accordance with results from this study, where SOSDE with higher concentration of gallic acid had a significantly lower AV compared to SOSDE with low concentration gallic acid ( $p<0.001$, Fig $2 i)$ however, SOSDE with higher concentration EDTA compared to lower concentration EDTA did not have lower AV in this study (Fig 2i).

Fig 2 ii presents the results of the effect of time of storage (days) of SOSDEs on AV and $P V$. There were no significant products $X$ days interaction effects for $A V$ or $P V$. There were no significant differences in PV over the 24 week ambient storage time ( $p=0.423$, Fig $2 \mathrm{ii})$. However, was a significant increase in AV over the 24-week storage period from 3.5 at week 0 to 6.2 at week 24 (Fig 2ii). It is clear that the AV increased more rapidly towards the end of the shelf life period (Fig 2ii).

In this study, the addition of EDTA had a higher retardation effect than the addition of gallic acid on the development of secondary oxidation products as measured by AV (Fig 2i). Boon, Xu, Yue and McClements (2008) also concluded that the addition of $100 \mu \mathrm{M}$ EDTA into an emulsion reduced lipid oxidation. EDTA retards oxidation by chelating iron, a pro-oxidant, to form FeEDTA (MacPhail, Patel, Bothwell, \& Lamparelli, 1994). A pH lower than 5 initiates the release of iron in oil-in-water emulsions which in turn decomposes pre-existing lipid hydroperoxides located at the oil-water interface (Thomsen, Jacobsen, \& Skibsted, 1999). The $\mathrm{pH}$ of SOSDEs in this study were measured at $3.24-3.32$ indicating the possibility of release of iron. With EDTA binding to the iron it retards the decomposition of pre-existing lipid hydroperoxides explaining why the SOSDE with EDTA had a lower AV (Fig 2i). Bou et al. (2011) found contradictory results for the effectiveness of EDTA but concluded that metal chelators such as EDTA are effective when used with anionic surfactants.

Comparing the results from sensory and chemical analyses for ambient stored SOSDEs, the higher concentration of gallic acid significantly decreased AV (Fig 2i) and rancid aroma (Fig 
1) compared to the lower. Addition of EDTA to SOSDE, lowered $(p<0.01)$ the AV (Fig 2i) as well as the cardboard aroma (Fig 1) compared to the addition of gallic acid to SOSDE. This supports previous findings that $\mathrm{AV}$ is a useful indicator of the actual state of rancidity in oil containing products (Hudson \& Gordon, 1999).

However, as a rule of thumb a product is only classified as rancid once the AV reaches higher than 10 (Rossel, 1999) and all products in ambient storage had AV measurements lower than 6.

\subsection{Sensory and chemical analyses of SOSDE with different levels of antioxidants and stored under accelerated shelf life conditions}

Fig 3 shows the aroma profiles of SOSDEs with low and high concentrations of antioxidants stored under accelerated shelf life conditions. Painty aroma was the only attribute that showed significant differences in detection between SOSDEs with high and low concentrations of gallic acid. Painty aroma in AccLoGal was perceived by $10 \%$ more panellists compared to AccHiGal.

Gallic acid applied to the SOSDE, lowered the detection of eggy and dairy significantly while it increased the detection of green and earthy aroma compared to SOSDEs with EDTA or no antioxidants. Metallic aroma was perceived by significantly more panellists in SOSDE with no antioxidants compared to SOSDE with gallic acid or EDTA. Cardboard aroma was significantly more noted in SOSDEs with EDTA compared to gallic acid. Plastic aroma was perceived by a very small proportion (less than $5 \%$ ) of panellists compared to other attributes (Fig 3). It is clear that plastic was not a differentiating attribute of lipid oxidation in this study. In other studies panellists had difficulty describing, rating or identifying specific rancidity related attributes. Serfert, Drusch and Schwarz (2010) and Böttcher, Steinhäuser and Drusch (2015) recommended to use overall rancidity measures, rather than specific descriptive attributes of rancidity during sensory analyses. 


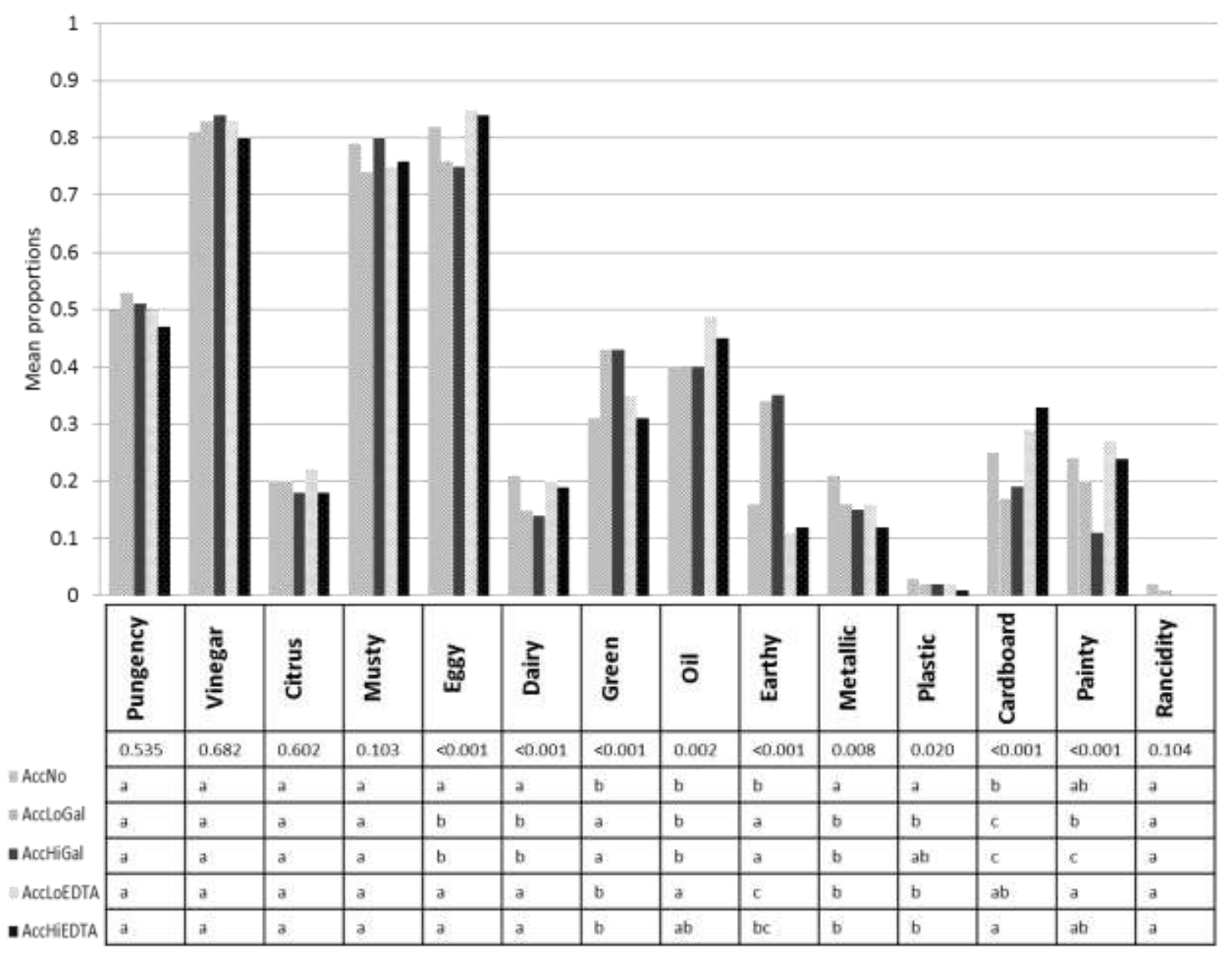

abc - for a specific aroma attribute, products with different letters were significantly different at the $95 \%$ confidence level

Fig 3. The effect of low and high concentrations of antioxidants or none added on aroma attributes for SOSDEs stored at accelerated conditions for 20 days with measurements taken every 24 hours (see Table 1 for detail of product code names)

To better understand how different concentrations of antioxidants gallic acid and EDTA influenced oxidation of SOSDEs, PV and AV were determined for each SOSDE every second day during accelerated storage (Fig 4). EDTA had a significant effect on the development of peroxides (measured as PV) as well as secondary peroxide products (measured as AV). PV and AV for AccHiEDTA were significantly lower (Fig 4i) compared to AccLoEDTA, indicating that the higher concentration of antioxidant had a greater effect on retarding lipid oxidation. This 
effect was also noted by Coppen, (1999) in a study with 200ppm and 2500ppm polymeric antioxidant in vegetable oil, where the higher concentration of polymeric antioxidant had a greater effect on oxidation. The reason why the AV of AccLoEDTA was significantly higher than AccNo is not clear. Contradictory to this, SOSDE with a low gallic acid concentration had a significantly lower AV compared to SOSDE with a high gallic acid concentration (Fig 4i). The SOSDEs containing gallic acid as the antioxidant had higher PV compared to the SOSDEs with EDTA as the antioxidant. PV showed no differences from day 1 to day 8 , with significant increases at day 14 and again at day 17 occurred (Fig 4 ii). As measurements were only taken until day 20, it is unknown if the PV would change after day 20. According to Robards, Kerr, and Patsalides, (1988) as the PV reaches a peak, an increase in AV could be expected as the peroxides decompose into volatiles. This expectation, was based on the negative correlation found between AV and PV in edible oil (Robards et al., 1988).

Comparing chemical analysis with sensory analysis for SOSDEs stored in accelerated conditions, EDTA addition to SOSDE lowered the PV but increased the detection of painty and cardboard aroma compared to SOSDE with gallic acid (Fig $4 \mathrm{I}$ and Fig 3). Similar results were seen with AccHiGal having a higher AV (Fig 4i) and a less detected painty aroma (Fig 3) compared to AccLoGal. This came as no surprise as peroxides and hydroperoxides as measured through PV were reported as hardly detectable (Hudson \& Gordon, 1999) and did not correlate with sensory data (Frankel, 1998). However their presence was a potential indicator of the development of rancid off-flavour as they inevitably break down to volatile compounds including but not limited to alcohols, aldehydes, ketones and carbonyl compounds that are responsible for the off flavours associated with rancidity (Cercaci, Rodriguez-Estrada, Lercker, \& Decker, 2007; Hudson \& Gordon, 1999).

In order to better understand oxidative stability of emulsions, sensory and chemical results from SOSDEs in ambient storage were compared to results from SOSDEs in accelerated storage. Reviewing the sensory results (Fig 1 and Fig 3) first, earthy aroma was perceived by 
i)

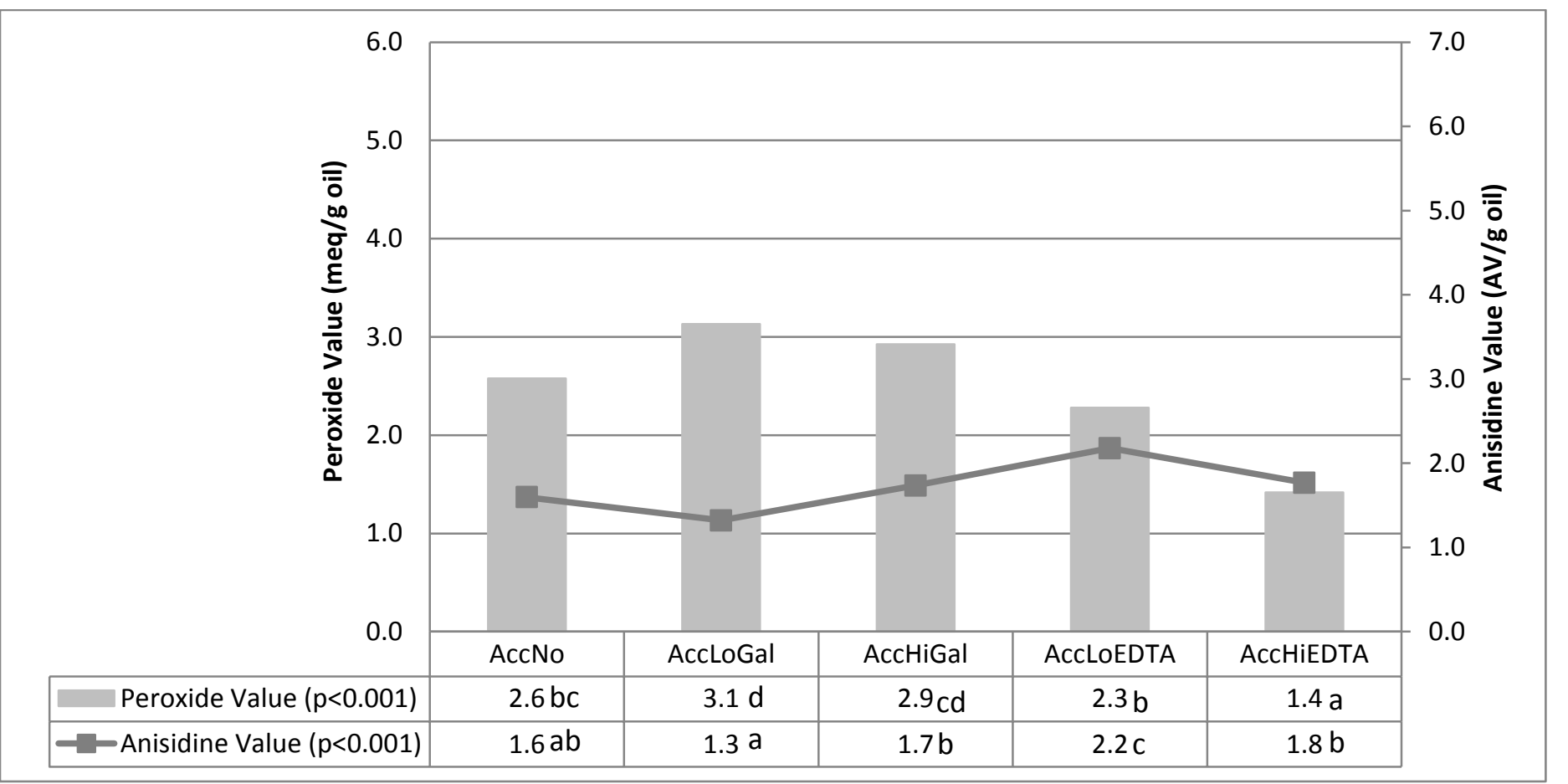

abcd - values among products with different letters indicate significant differences at $99 \%$ confidence level

ii)

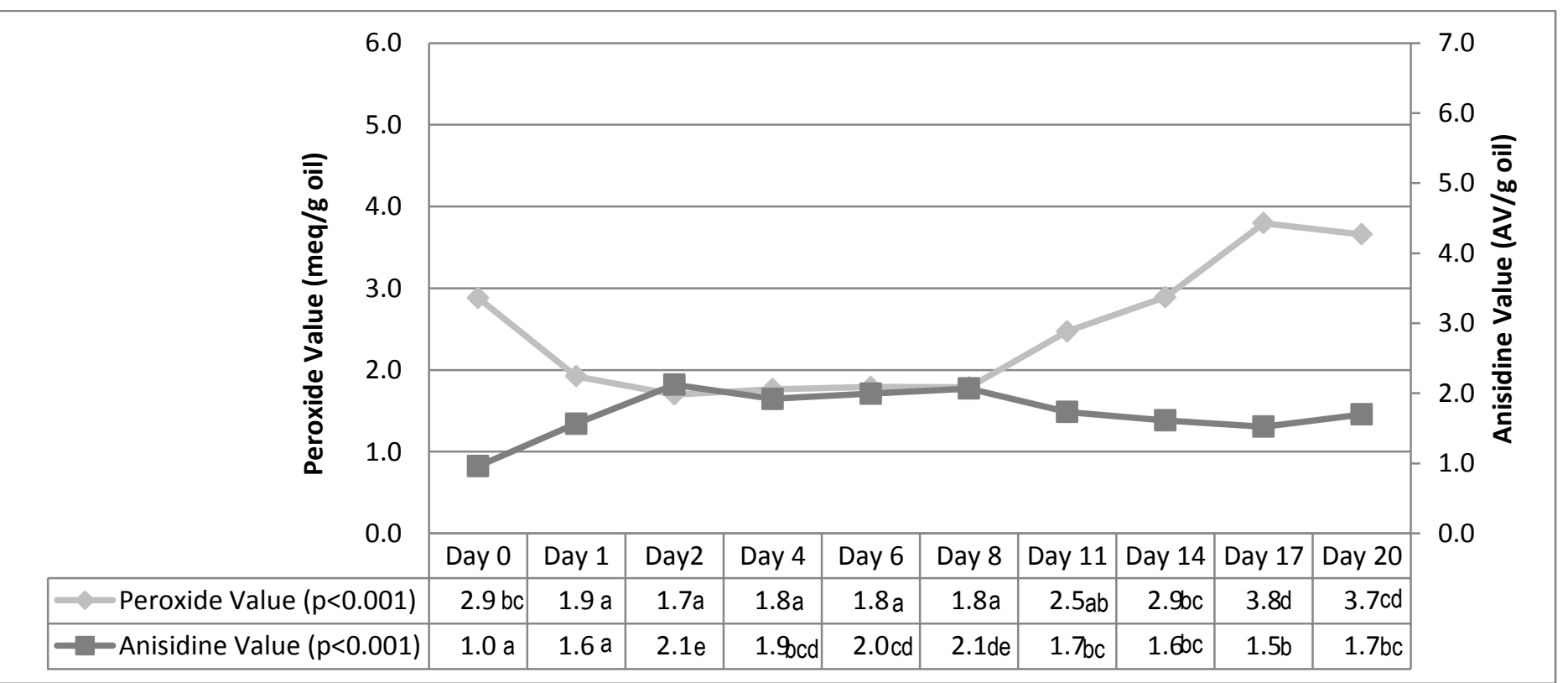

abcde - values in a row with different letters indicate significant differences at $99 \%$ confidence level

Fig 4. Chemical analyses (Peroxide Value and Anisidine Value) for oil extracted from SOSDEs stored under accelerated shelf life conditions illustrated for (i) different concentrations of EDTA and gallic acid and (ii) 20 day time period. 
significantly more panellists in SOSDE with gallic acid compared to SOSDE with EDTA across both storage conditions. The earthy aroma may possible be associated with the mere presence of gallic acid in the SOSDE rather than lipid oxidation. However, no association of earthy flavour or aroma with gallic acid could be found in literature. There were no other similar patterns observed between the different antioxidants in the two storage conditions.

Upon comparison of chemical analyses results for SOSDEs in ambient storage (Fig 2) with SOSDEs in accelerated storage (Fig 4), interesting findings were made. With pro-oxidants $\mathrm{FeSO}_{4}$ and ascorbic acid added to SOSDEs it was expected that PV for SOSDEs in accelerated storage would be higher than PV for SOSDE in ambient storage (Branco et al., 2011). However the average PVs for SOSDEs from accelerated storage was lower $1.42-3.13 \mathrm{meq} / \mathrm{g}$ (Fig 4i), compared to ambient stored SOSDEs which ranged from $5.20-5.74 \mathrm{meq} / \mathrm{g}$ (Fig 2i). This was inconsistent to findings by Jayasinghe, Gotoh and Wada (2013) who demonstrated that the presence of a transition metal in oil-in-water emulsions, led to higher formation rates of hydroperoxides (measured by PV) which was further accelerated by the addition of ascorbic acid as it reduced the $\mathrm{Fe}^{3+}$. Jayasinghe, et al. (2013) further noted that the hydroperoxides are unstable compounds that are easily decomposed. In fact, the transition metal in reduced state $\mathrm{Fe}^{2+}$, as was added here, is very efficient at decomposing hydroperoxides (McClements \& Decker, 2008) which would explain the lower PVs.Changes in AV over time were inconsistent between accelerated and ambient stored SOSDEs. AccHiGal had higher AV compared to AccLoGal (Fig 4i), while AmbHiGal had lower AV than AmbLoGal (Fig 2i). The same was found for SOSDEs with EDTA, where AccHiEDTA had lower AV than AccLoEDTA (Fig 4i), with no differences between AmbHiEDTA and AmbLoEDTA SOSDEs (Fig 2i). This may be due to the fact the SOSDEs in both storage systems were oxidised, but through different mechanisms due 
to the addition of pro-oxidants. The result obtained with the specific accelerated model can be attributed to the fact that Fe2+ reacted with EDTA, a potent metal chelator.

\section{CONCLUSIONS}

The effects of different concentrations of a natural antioxidant (gallic acid) and a synthetic antioxidant (EDTA) on orthonasal aroma, AV and PV of a SOSDE stored under ambient conditions $\left(25^{\circ} \mathrm{C}\right)$ and an accelerated storage model incorporating a higher temperature (32.2 $\left.{ }^{\circ} \mathrm{C}\right)$ with $\mathrm{Fe} 2+$ and ascorbic acid added, was determined.

At ambient conditions, the addition of EDTA to the SOSDE retarded lipid oxidation as measured through aroma and chemical analysis (PV and AV). However, when the SOSDE was stored under accelerated conditions, addition of a low concentration of EDTA led to a higher AV while a high concentration led to a lower AV compared to the SOSDE with no antioxidants added. . Increased lipid oxidation retardation was noted for higher concentrations of gallic acid by AV analysis and by rancid aroma detection during ambient shelf life. Sensory differences between products with high and low concentrations of antioxidants were more apparent during the ambient storage while PV and AV differences differentiated samples more clearly when stored under accelerated conditions.

These results suggest that: 1) the accelerated storage model used in this study (incorporating storage at $32.2^{\circ} \mathrm{C}$ with $\mathrm{Fe}^{2+}$ and ascorbic acid added) is more suitable for SOSDEs with metal chelator antioxidants such as EDTA, rather than free radical scavenging antioxidants such as gallic acid, 2) for this SOSDE, PV and AV compared to orthonasal aroma analysis with a sensory panel are more discriminating oxidation markers when using this accelerated model for 20 days of storage. Orthonasal aroma analysis may not be sensitive enough to detect small changes in formation of lipid oxidation products and therefore full sensory analysis including retronasal aroma and taste perception is recommended for future studies on lipid oxidation in 
oil-in-water emulsions. Further to this it is also not known what level of aroma attributes constitute unacceptable quality and therefore end of shelf life. Verification of the end of shelf life as a function of product rejection by consumers is needed to validate measurements by sensory panel and chemical oxidation markers.

3) PV, AV and aroma of SOSDEs stored in accelerated conditions for 20 days as used in this study, do not clearly predict results for ambient stored SOSDEs.

It is recommended that for future shelf life research, shelf stable oil-in-water emulsions should be stored for longer than six months at ambient storage conditions to effectively monitor the development of lipid oxidation in the presence of antioxidants.

\section{ACKNOWLEDGEMENTS}

We would like to thank Dr.Hamed Faridi, Chief Science Officer, McCormick \& Co., Inc for supporting this research and Marie Smith for assistance with statistical analyses.

\section{REFERENCES}

Alamed, J., Mcclements, D. J., \& Decker, E. A. (2006). Influence of heat processing and calcium ions on the ability of EDTA to inhibit lipid oxidation in oil-in-water emulsions containing omega-3 fatty acids. Food Chemistry, 95, 585-590.

AOCS. 1999. Official Methods and Recommended Practices of the American Oils Chemists Society, 5th Ed., Champaign: AOCS Press.

Arancibia, C., Jublot, L., Costell, E., \& Bayarri, S. (2011). Flavor release and sensory characteristics of o/w emulsions. Influences of composition, microstructure and rheological behaviour. Food Research International, 44, 1632-1641. 
Asnaashari, M., Farhoosh, R., \& Sharif, A. (2014). Antioxidant activity of gallic acid and methyl gallate in triacylglycerols of Kilka fish oil and its oil-in-water emulsion. Food Chemistry, 159, 439-444.

Boon, C., Xu, Z., Yue, X., \& McClements, D. J. (2008). Factors affecting lycopene oxidation in oil-in-water emulsions. Journal of Agriculture and Food Chemistry, 56, 1408-1414.

Böttcher, S., Steinhäuser, U., \& Drusch, S. (2015). Off-flavour masking of secondary lipid oxidation products by pea dextrin. Food Chemistry, 169, 492-498.

Bou, R., Boon, C., Kweku, A.,Hidalgo, D., \& Decker, E. A. (2011). Effect of different antioxidants on lycopene degradation in oil-in-water emulsion. European Journal of Lipid Science and Technology, 113, 724-729.

Branco, G. F., Rodrigues, M. I., Gioielli, L. A., \& Castro, I. A. (2011). Effect of the simultaneous interaction among ascorbic acid, iron and $\mathrm{pH}$ on the oxidative stability of oil-in-water emulsions. Journal of Agriculture and Food Chemistry, 59, 12183-12192.

Cercaci, L., Rodriguez-Estrada, M. T., Lercker, G., \& Decker, E. A. (2007). Phytosterol oxidation in oil-inwater emulsions and bulk oil. Food Chemistry 102, 161-167.

Chen, B., McClements, D. J., \& Decker, E. A. (2011). Minor components in food oils: Review of their roles on lipid oxidation chemistry in bulk oils and emulsions. Critical Reviews in Food Science and Nutrition, 51(10), 901-916.

Coppen, P. (1999). The use of antioxidants. In J. Allen \& R. Hamilton (Eds.), Rancidity in Foods (3rd ed.). Gaitersburg, MD, USA: Aspen Publisher.

Córdova, A., Quezada, C., \& Saavedra, J. (2011). A MALST method comparison over univariate kinetic modeling for determination of shelf-life in cereal snack of dried apples. Procedia Food Science, 1, 1045-1050.

Corrigan, V., Hedderley, D., \& Harvey, W. (2012). Modeling the shelf life of fruit-filled snack bars using survival analysis and sensory profiling techniques. Journal of Sensory Studies, 27(6), 403-416.

Frankel, E. N. (1998). Lipid Oxidation. Dundee, Scotland:The Oily Press. 
Hamilton, R. J. (1999). The chemistry of rancidity in foods. In J. C. Allen \& R. J. Hamilton (Eds.), Rancidity in foods (3rd ed.). Gaitersburg, MD, USA: Aspen Publisher.

Hartvigsen, K., Lund, P., Hansen, L. F., \& Holmer, C. (2000). Dynamic headspace gas chromatography/mass spectrometry characterization of volatiles produced in fish oil enriched mayonnaise during storage. Journal of Agriculture and Food Chemistry, 48, 4858-4867.

Hudson, B. J. F., \& Gordon, M. H. (1999). Evaluation of oxidative rancidity techniques. In J. C. Allen \& R. J. Hamilton (Eds.), Rancidity in foods (3rd ed.). Gaitersburg, MD, USA: Aspen Publisher.

Jacobsen, C. (1999). Sensory impact of lipid oxidation in complex food systems. European Journal of Lipid Science and Technology, 101(12), 484-492.

Jayasinghe, C., Gotoh, N., \& Wada, S. (2013). Pro-oxidant / antioxidant behaviours of ascorbic acid, tocopherol, and plant extracts in n-3 highly unsaturated fatty acid rich oil-in-water emulsions. Food Chemistry, 141(3), 3077-3084.

Lerma-García, M. J., Cerretani, L., Cevoli, C., Simó-Alfonso, E. F., Bendini, A., \& Toschi, T. G. (2010). Use of electronic nose to determine defect percentage in oils. Comparison with sensory panel results. Sensors and Actuators B: Chemical, 147, 283-289.

Lewis, M. J, \& Heppel, N. J. (2000). Continues thermal processing of foods, pasteurization and UHT sterilization. Gaitersburg, MD, USA: Aspen Publisher.

MacPhail, A. P., Patel, R. C, Bothwell, T. H, \& Lamparelli, R. D. (1994). EDTA and the absorption of iron from food. American Journal of Clinical Nutrition, 59, 644-648.

McClements, D. J. \& Decker, E. A. (2008). Lipids. In: Fennema's Food Chemistry, 4th ed., pp. 155-216. Srinivasan, D., Parkin, K. L., and Fennema, O. R., Eds., CRC Press/Taylor \& Francis, Boca Raton, FL.

Meilgaard, M. C., Civille, G. V., \& Carr, B. T. (2007). Sensory evaluation techniques. New York, NY, USA: CRC Press.

Payne, R. (2012). Introduction to GenStat for Windows. Retrieved from http://www.genstat.co.uk 
Richards, M., De Kock, H. L., \& Buys, E. M. (2014). Multivariate accelerated shelf life test of low fat UHT milk. International Dairy Journal, 36, 38-45.

Robards, K., Kerr, A. F., \& Patsalides, E. (1988). Rancidity and its measurement in edible oils and snack foods. Analyst, 113, 213-224.

Rodríguez-Rojo, S., Visentin, A., Maestri, D., \& Cocero, M. J. (2012). Assisted extraction of rosemary antioxidants with green solvents. Journal of Food Engineering, 109, 98-103.

Rossel, J. B. (1999). Measurement of rancidity. In J.C. Allen \& R. J. Hamilton (Eds.), Rancidity in foods (3rd ed.). Gaitersburg, MD, USA: Aspen Publisher.

Serfert, Y., Drusch, S., \& Schwarz, K. (2010). Sensory odour profiling and lipid oxidation status of fish oil and microencapsulated fish oil. Food Chemistry, 123(4), 968-975.

Shanta, N. C., \& Decker, E. A. (1994). Rapid, sensitive, iron-based spectrophotometric methods for determination of peroxide values of food lipids. Journal of Association of Official Analytical Chemists International, 77(2), 421-424.

Thomsen, M. K., Jacobsen, C., \& Skibsted, L. H. (2000). Mechanisms of initiation of oxidation in mayonaise enriched with fish oil as studied by electron spin resonance spectroscopy. European Food Research and Technology, 211, 381-386

Zimmerman, D. W. (2000). Statistical significance levels of nonparametric tests biased by heterogeneous variances of treatment groups. Journal of General Psychology, 127(4), 354-364. 